Research Article

Vieri Benci, Lorenzo Luperi Baglini and Marco Squassina*

\title{
Generalized solutions of variational problems and applications
}

https://doi.org/10.1515/anona-2018-0146

Received June 27, 2018; accepted June 27, 2018

\begin{abstract}
Ultrafunctions are a particular class of generalized functions defined on a hyperreal field $\mathbb{R}^{*} \supset \mathbb{R}$ that allow to solve variational problems with no classical solutions. We recall the construction of ultrafunctions and we study the relationships between these generalized solutions and classical minimizing sequences. Finally, we study some examples to highlight the potential of this approach.
\end{abstract}

Keywords: Ultrafunctions, non-Archimedean mathematics, nonstandard analysis, delta function

MSC 2010: 03H05, 26E35, 28E05, 46S20

\section{Introduction}

It is nowadays very well known that, in many circumstances, the needs of a theory require the introduction of generalized functions. Among people working in partial differential equations, the theory of distributions of L. Schwartz is the most commonly used, but other notions of generalized functions have been introduced, e.g. by J. F. Colombeau [15] and M. Sato [21, 22]. Many notions of generalized functions are based on nonArchimedean mathematics, namely mathematics handling infinite and/or infinitesimal quantities. Such an approach presents several positive features, the main probably being the possibility of treating distributions as non-Archimedean set-theoretical functions (under the limitations imposed by Schwartz' result). This allows to easily introduce nonlinear concepts, such as products, into distribution theory. Moreover, a theory which includes infinitesimals and infinite quantities makes it possible to easily construct new models, allowing in this way to study several problems which are difficult even to formalize in classical mathematics. This has led to applications in various field, including several topics in analysis, geometry and mathematical physic (see e.g. $[17,19]$ for an overview in the case of Colombeau functions and their recent extension, called generalized smooth functions).

In this paper we deal with ultrafunctions, which are a kind of generalized functions that have been introduced recently in [1] and developed in [2, 4-11].

Ultrafunctions are a particular case of non-Archimedean generalized functions that are based on the hyperreal field $\mathbb{R}^{*}$, namely the numerical field on which nonstandard analysis is based. (We refer to Keisler [16] for a very clear exposition of nonstandard analysis.) No prior knowledge of nonstandard analysis is requested to read this paper: we will introduce all the nonstandard notions that we need via a new notion of limit, called $\Lambda$-limit (see [10] for a complete introduction to this notion and its relationships with the usual nonstandard analysis). The main peculiarity of this notion of limit is that it allows us to make a very limited use of formal logic, in contrast with most usual nonstandard analysis introductions.

Vieri Benci, Dipartimento di Matematica, Università degli Studi di Pisa, Via F. Buonarroti 1/c, 56127 Pisa; and Centro Linceo interdisciplinare Beniamino Segre, Palazzo Corsini - Via della Lungara 10, 00165 Roma, Italy, e-mail: benci@dma.unipi.it Lorenzo Luperi Baglini, Faculty of Mathematics, University of Vienna, Oskar-Morgenstern-Platz 1, 1090 Wien, Austria, e-mail: lorenzo.luperi.baglini@univie.ac.at. https://orcid.org/0000-0002-0559-0770

*Corresponding author: Marco Squassina, Dipartimento di Matematica e Fisica, Università Cattolica del Sacro Cuore, Via dei Musei 41, 25121 Brescia, Italy, e-mail: marco.squassina@unicatt.it 
Apart from being framed in a non-Archimedean setting, ultrafunctions have other peculiar properties that will be introduced and used in the following:

- every ultrafunction can be split uniquely (in a sense that will be precised in Section 3.2) as the sum of a classical function and a purely non-Archimedean part;

- ultrafunctions extend distributions, in the sense that every distribution can be identified with an ultrafunction; in particular, this allows to perform nonlinear operations with distributions;

- although being generalized functions, ultrafunctions share many properties of $e^{1}$ functions, like e.g. Gauss' divergence theorem.

Our goal is to introduce all the aforementioned properties of ultrafunctions, so to be able to explain how they can be used to solve certain classical problems that do not have classical solutions; in particular, we will concentrate on singular problems arising in calculus of variations and in relevant applications (see e.g. [17] and references therein for other approaches to these problems based on different notions of generalized functions).

The paper is organized as follows: in Section 2, we introduce the notion of $\Lambda$-limit, and we explain how to use it to construct all the non-Archimedean tools that are needed in the rest of the paper, in particular, how to construct the non-Archimedean field extension $\mathbb{R}^{*}$ of $\mathbb{R}$ and what the notion of "hyperfinite" means. In Section 3 we define ultrafunctions, and we explain how to extend derivatives and integrals to them. All the properties of ultrafunctions needed later on are introduced in this section: we show how to split an ultrafunction as the sum of a standard and a purely non-Archimedean part, how to extend Gauss' divergence theorem and how to identify distributions with certain ultrafunctions. In Section 4, we present the main results of the paper, namely, we show that a very large class of classical problems admits generalized ultrafunction solutions. We study the main properties of these generalized solutions, concentrating in particular on the relationships between ultrafunction solutions and classical minimizing sequences for variational problems. Finally, in Section 5, we present two examples of applications of our methods: the first is the study of a variational problem related to the sign-perturbation of potentials, the second is a singular variation problem related to sign-changing boundary conditions.

The first part of this paper contains some overlap with other papers on ultrafunctions, but this fact is necessary to make it self-contained and to make the reader comfortable with it.

\subsection{Notations}

If $X$ is a set and $\Omega$ is a subset of $\mathbb{R}^{N}$, then

- $\quad \mathcal{P}(X)$ denotes the power set of $X$ and $\mathcal{P}_{\text {fin }}(X)$ denotes the family of finite subsets of $X$;

- $\mathfrak{F}(X, Y)$ denotes the set of all functions from $X$ to $Y$ and $\mathfrak{F}(\Omega)=\mathfrak{F}(\Omega, \mathbb{R})$;

- $\mathcal{C}(\Omega)$ denotes the set of continuous functions defined on $\Omega \subset \mathbb{R}^{N}$;

- $\quad e^{k}(\Omega)$ denotes the set of functions defined on $\Omega \subset \mathbb{R}^{N}$ which have continuous derivatives up to the order $k$ (sometimes we will use the notation $\mathscr{C}^{0}(\Omega)$ instead of $\mathscr{C}(\Omega)$ );

- $H^{k, p}(\Omega)$ denotes the usual Sobolev space of functions defined on $\Omega \subset \mathbb{R}^{N}$;

- if $W(\Omega)$ is any function space, then $W_{c}(\Omega)$ will denote the function space of functions in $W(\Omega)$ having compact support;

- $\mathcal{C}_{0}(\Omega \cup \Xi), \Xi \subseteq \partial \Omega$, denotes the set of continuous functions in $\mathcal{C}(\Omega \cup \Xi)$ which vanish for $x \in \Xi$;

- $\mathcal{D}(\Omega)$ denotes the set of the infinitely differentiable functions with compact support defined on $\Omega \subset \mathbb{R}^{N}$; $\mathcal{D}^{\prime}(\Omega)$ denotes the topological dual of $\mathcal{D}(\Omega)$, namely the set of distributions on $\Omega$;

- if $A \subset X$ is a set, then $\chi_{A}$ denotes the characteristic function of $A$;

- $\quad \operatorname{supp}(f)=\operatorname{supp}^{*}(f)$ where supp is the usual notion of support of a function or a distribution;

- $\operatorname{mon}(x)=\left\{y \in\left(\mathbb{R}^{N}\right)^{*}: x \sim y\right\}$, where $x \sim y$ means that $x-y$ is infinitesimal;

- $\quad \forall^{\text {a.e. }} x \in X$ means "for almost every $x \in X$ ";

- if $a, b \in \mathbb{R}^{*}$, then

- $[a, b]_{\mathbb{R}^{*}}=\left\{x \in \mathbb{R}^{*}: a \leq x \leq b\right\}$,

- $\quad(a, b)_{\mathbb{R}^{*}}=\left\{x \in \mathbb{R}^{*}: a<x<b\right\} ;$ 
- if $W$ is a generic function space, its topological dual will be denoted by $W^{\prime}$ and the pairing by $\langle\cdot, \cdot\rangle_{W}$;

- $\quad$ if $E$ is any set, then $|E|$ will denote its cardinality.

\section{$2 \Lambda$-theory}

In this section, we present the basic notions of Non-Archimedean Mathematics (sometimes abbreviated as NAM) and of Nonstandard Analysis (sometimes abbreviated as NSA) following a method inspired by [3] (see also [1] and [4]). When we talk about NSA, we mean the NSA in the sense of Robinson (see [20]), and not the internal set theory developed by Nelson in [18].

\subsection{Non-Archimedean fields}

Here, we recall the basic definitions and facts regarding non-Archimedean fields. In the following, $\mathbb{K}$ will denote a totally ordered infinite field. We recall that such a field contains (a copy of) the rational numbers. Its elements will be called numbers.

Definition 2.1. Let $\mathbb{K}$ be an ordered field. Let $\xi \in \mathbb{K}$. We say that

- $\quad \xi$ is infinitesimal if, for all positive $n \in \mathbb{N},|\xi|<\frac{1}{n}$;

- $\quad \xi$ is finite if there exists $n \in \mathbb{N}$ such that $|\xi|<n$;

- $\quad \xi$ is infinite if, for all $n \in \mathbb{N},|\xi|>n$ (equivalently, if $\xi$ is not finite).

Definition 2.2. An ordered field $\mathbb{K}$ is called non-Archimedean if it contains an infinitesimal $\xi \neq 0$.

It is easily seen that infinitesimal numbers are actually finite, that the inverse of an infinite number is a nonzero infinitesimal number, and that the inverse of a nonzero infinitesimal number is infinite.

Definition 2.3. A superreal field is an ordered field $\mathbb{K}$ that properly extends $\mathbb{R}$.

It is easy to show, due to the completeness of $\mathbb{R}$, that there are nonzero infinitesimal numbers and infinite numbers in any superreal field. Infinitesimal numbers can be used to formalize a new notion of closeness, according to the following:

Definition 2.4. We say that two numbers $\xi, \zeta \in \mathbb{K}$ are infinitely close if $\xi-\zeta$ is infinitesimal. In this case, we write $\xi \sim \zeta$.

Clearly, the relation $\sim$ of infinite closeness is an equivalence relation and we have the following:

Theorem 2.5. If $\mathbb{K}$ is a totally ordered superreal field, every finite number $\xi \in \mathbb{K}$ is infinitely close to a unique real number $r \sim \xi$, called the standard part of $\xi$.

Given a finite number $\xi$, we denote its standard part by st $(\xi)$, and we put $\operatorname{st}(\xi)= \pm \infty$ if $\xi \in \mathbb{K}$ is a positive (negative) infinite number. In Definition 2.16, we will see how the notion of standard part can be generalized to any Hausdorff topological space.

Definition 2.6. Let $\mathbb{K}$ be a superreal field and $\xi \in \mathbb{K}$ a number. The monad of $\xi$ is the set of all numbers that are infinitely close to it,

$$
\mathfrak{m o n}(\xi):=\{\zeta \in \mathbb{K}: \xi \sim \zeta\}
$$

\subsection{The $\Lambda$-limit}

Let $U$ be an infinite set of cardinality bigger than the continuum, and let $\mathfrak{L}=\mathscr{P}_{\text {fin }}(U)$ be the family of finite subsets of $U$. 
Notice that $(\mathfrak{L}, \subseteq)$ is a directed set. We add to $\mathfrak{L}$ a point at infinity $\Lambda \notin \mathfrak{L}$, and we define the following family of neighborhoods of $\Lambda$ :

$$
\{\{\Lambda\} \cup Q: Q \in \mathcal{U}\},
$$

where $\mathcal{U}$ is a fine ultrafilter on $\mathfrak{L}$, namely a filter such that

- for every $A, B \subseteq \mathfrak{L}$, if $A \cup B=\mathfrak{L}$, then $A \in \mathcal{U}$ or $B \in \mathcal{U}$;

- for every $\lambda \in \mathfrak{L}$, the set $Q(\lambda):=\{\mu \in \mathfrak{L}: \lambda \sqsubseteq \mu\} \in \mathcal{U}$.

We will refer to the elements of $\mathcal{U}$ as qualified sets. A function $\varphi: \mathfrak{L} \rightarrow E$, defined on a directed set $E$, is called net (with values in $E$ ). If $\varphi(\lambda)$ is a real net, we have that

$$
\lim _{\lambda \rightarrow \Lambda} \varphi(\lambda)=L
$$

if, and only if, for every $\varepsilon>0$, there exists $Q \in \mathcal{U}$ such that $|\varphi(\lambda)-L|<\varepsilon$ for all $\lambda \in Q$.

As usual, if a property $P(\lambda)$ is satisfied by any $\lambda$ in a neighborhood of $\Lambda$, we will say that it is eventually satisfied.

Proposition 2.7. If the net $\varphi(\lambda)$ takes values in a compact set $K$, then it is a converging net.

Proof. Suppose that the net $\varphi(\lambda)$ has a converging subnet to $L \in \mathbb{R}$. We fix $\varepsilon>0$ arbitrarily, and we have to prove that $Q_{\varepsilon} \in \mathcal{U}$, where

$$
Q_{\varepsilon}=\{\lambda \in \mathfrak{L}:|\varphi(\lambda)-L|<\varepsilon\} .
$$

We argue indirectly, and we assume that $Q_{\varepsilon} \notin \mathcal{U}$. Then, by the definition of ultrafilter, $N=\mathfrak{L} \backslash Q_{\varepsilon} \in \mathcal{U}$, and hence,

$$
|\varphi(\lambda)-L| \geq \varepsilon \text { for all } \lambda \in N .
$$

This contradicts the fact that $\varphi_{\lambda}$ has a subnet which converges to $L$.

Proposition 2.8. Assume that $\varphi: \mathfrak{L} \rightarrow E$, where $E$ is a first countable topological space; then if

$$
\lim _{\lambda \rightarrow \Lambda} \varphi(\lambda)=x_{0},
$$

there exists a sequence $\left\{\lambda_{n}\right\}$ in $\mathfrak{L}$ such that

$$
\lim _{n \rightarrow \infty} \varphi\left(\lambda_{n}\right)=x_{0}
$$

We refer to the sequence $\varphi_{n}:=\varphi\left(\lambda_{n}\right)$ as a subnet of $\varphi(\lambda)$.

Proof. Let $\left\{A_{n}: n \in \mathbb{N}\right\}$ be a countable basis of open neighborhoods of $x_{0}$. For every $n \in \mathbb{N}$, the set

$$
I_{n}:=\left\{\lambda \in \mathfrak{L}: \varphi(\lambda) \in A_{n}\right\}
$$

is qualified. Hence, $J_{n}:=\bigcap_{j \leq n} I_{j} \neq \emptyset$. Let $\lambda_{n} \in J_{n}$. Then the sequence $\left\{\lambda_{n}\right\}_{n \in \mathbb{N}}$ has trivially the desired property: for every $n \in \mathbb{N}$, for every $m \geq n$, we have that $\varphi\left(\lambda_{m}\right) \in A_{n}$.

Example 2.9. Let $\varphi: \mathfrak{L} \rightarrow V$ be a net with values in a bounded subset of a reflexive Banach space equipped with the weak topology; then

$$
v:=\lim _{\lambda \rightarrow \Lambda} \varphi(\lambda)
$$

is uniquely defined, and there exists a sequence $n \mapsto \varphi\left(\lambda_{n}\right)$ which converges to $v$.

Definition 2.10. The set of the hyperreal numbers $\mathbb{R}^{*} \supset \mathbb{R}$ is a set equipped with a topology $\tau$ such that

- every net $\varphi: \mathfrak{L} \rightarrow \mathbb{R}$ has a unique limit in $\mathbb{R}^{*}$, if $\mathfrak{L}$ and $\mathbb{R}^{*}$ are equipped with the $\Lambda$ and the $\tau$ topology, respectively;

- $\quad \mathbb{R}^{*}$ is the closure of $\mathbb{R}$ with respect to the topology $\tau$;

- $\tau$ is the coarsest topology which satisfies the first property.

The existence of such $\mathbb{R}^{*}$ is a well-known fact in NSA. The limit $\xi \in \mathbb{R}^{*}$ of a net $\varphi: \mathfrak{L} \rightarrow \mathbb{R}$ with respect to the $\tau$ topology, following [1], is called the $\Lambda$-limit of $\varphi$, and the following notation will be used:

$$
\xi=\lim _{\lambda \uparrow \Lambda} \varphi(\lambda) ;
$$


namely, we shall use the up-arrow " " to remind that the target space is equipped with the topology $\tau$. Given

$$
\xi:=\lim _{\lambda \uparrow \Lambda} \varphi(\lambda), \quad \eta:=\lim _{\lambda \uparrow \Lambda} \psi(\lambda),
$$

we set

$$
\begin{aligned}
\xi+\eta & :=\lim _{\lambda \uparrow \Lambda}(\varphi(\lambda)+\psi(\lambda)), \\
\xi \cdot \eta & :=\lim _{\lambda \uparrow \Lambda}(\varphi(\lambda) \cdot \psi(\lambda)) .
\end{aligned}
$$

Then the following well-known theorem holds:

Theorem 2.11. The definitions (2.2) and (2.3) are well posed and $\mathbb{R}^{*}$, equipped with these operations, is a nonArchimedean field.

Remark 2.12. We observe that the field of hyperreal numbers is defined as a sort of completion of real numbers. In fact, $\mathbb{R}^{*}$ is isomorphic to the ultrapower $\mathbb{R}^{\mathfrak{L}} / \mathfrak{I}$, where $\mathfrak{I}=\{\varphi: \mathfrak{L} \rightarrow \mathbb{R} \mid \varphi(\lambda)=0$ eventually $\}$. The isomorphism resembles the classical one between real numbers and equivalence classes of Cauchy sequences. This method is surely known to the reader for the construction of the real numbers starting from the rationals.

\subsection{Natural extension of sets and functions}

To develop applications, we need to extend the notion of $\Lambda$-limit to sets and functions (but also to differential and integral operators). This will allow to enlarge the notions of variational problem and of variational solution.

$\Lambda$-limits of bounded nets of mathematical objects in $V_{\infty}(\mathbb{R})$ can be defined by induction (a net $\varphi: \mathfrak{L} \rightarrow$ $V_{\infty}(\mathbb{R})$ is called bounded, if there exists $n \in \mathbb{N}$ such that, for all $\lambda \in \mathfrak{L}, \varphi(\lambda) \in V_{n}(\mathbb{R})$ ). To do this, consider a net

$$
\varphi: \mathfrak{L} \rightarrow V_{n}(\mathbb{R}) .
$$

Definition 2.13. For $n=0, \lim _{\lambda \uparrow \Lambda} \varphi(\lambda)$ is defined by (2.1); so by induction, we may assume that the limit is defined for $n-1$, and we define it for the net (2.4) as follows:

$$
\lim _{\lambda \uparrow \Lambda} \varphi(\lambda)=\left\{\lim _{\lambda \uparrow \Lambda} \psi(\lambda): \psi: \mathfrak{L} \rightarrow V_{n-1}(\mathbb{R}), \text { for all } \lambda \in \mathfrak{L}, \psi(\lambda) \in \varphi(\lambda)\right\} .
$$

A mathematical entity (number, set, function or relation) which is the $\Lambda$-limit of a net is called internal.

Definition 2.14. If for all $\lambda \in \mathfrak{L}, E_{\lambda}=E \in V_{\infty}(\mathbb{R})$, we set $\lim _{\lambda \uparrow \Lambda} E_{\lambda}=E^{*}$, namely

$$
E^{*}:=\left\{\lim _{\lambda \uparrow \Lambda} \psi(\lambda): \psi(\lambda) \in E\right\}
$$

$E^{*}$ is called the natural extension of $E$.

Notice that, while the $\Lambda$-limit of a constant sequence of numbers gives this number itself, a constant sequence of sets gives a larger set, namely $E^{*}$. In general, the inclusion $E \subseteq E^{*}$ is proper.

Given any set $E$, we can associate to it two sets: its natural extension $E^{*}$ and the set $E^{\sigma}$, where

$$
E^{\sigma}:=\left\{X^{*}: X \in E\right\} .
$$

Clearly $E^{\sigma}$ is a copy of $E$; however, it might be different as set since, in general, $X^{*} \neq X$.

Remark 2.15. If $\varphi: \mathfrak{L} \rightarrow X$ is a net with value in a topological space, we have the usual limit

$$
\lim _{\lambda \rightarrow \Lambda} \varphi(\lambda)
$$

which, by Proposition 2.7, always exists in the Alexandrov compactification $X \cup\{\infty\}$. Moreover, we have the $\Lambda$-limit, that always exists and it is an element of $X^{*}$. In addition, the $\Lambda$-limit of a net is in $X^{\sigma}$ if, and only if, $\varphi$ is eventually constant. If $X=\mathbb{R}$, and both limits exist, then

$$
\lim _{\lambda \rightarrow \Lambda} \varphi(\lambda)=\operatorname{st}\left(\lim _{\lambda \uparrow \Lambda} \varphi(\lambda)\right) .
$$


The above equation suggests the following definition.

Definition 2.16. If $X$ is topological space equipped with a Hausdorff topology, and $\xi \in X^{*}$, we set

$$
\operatorname{St}_{X}(\xi)=\lim _{\lambda \rightarrow \Lambda} \varphi(\lambda)
$$

if there is a net $\varphi: \mathfrak{L} \rightarrow X$ converging in the topology of $X$, and such that

$$
\xi=\lim _{\lambda \uparrow \Lambda} \varphi(\lambda),
$$

and $\operatorname{St}_{X}(\xi)=\infty$ otherwise.

By the above definition, we have that

$$
\lim _{\lambda \rightarrow \Lambda} \varphi(\lambda)=\operatorname{St}_{X}\left(\lim _{\lambda \uparrow \Lambda} \varphi(\lambda)\right)
$$

Definition 2.17. Let

$$
f_{\lambda}: E_{\lambda} \rightarrow \mathbb{R}, \quad \lambda \in \mathfrak{L},
$$

be a net of functions. We define a function

$$
f:\left(\lim _{\lambda \uparrow \Lambda} E_{\lambda}\right) \rightarrow \mathbb{R}^{*}
$$

as follows: for every $\xi \in\left(\lim _{\lambda \uparrow \Lambda} E_{\lambda}\right)$, we set

$$
f(\xi):=\lim _{\lambda \uparrow \Lambda} f_{\lambda}(\psi(\lambda)),
$$

where $\psi(\lambda)$ is a net of numbers such that

$$
\psi(\lambda) \in E_{\lambda} \quad \text { and } \quad \lim _{\lambda \uparrow \Lambda} \psi(\lambda)=\xi .
$$

A function which is a $\Lambda$-limit is called internal. In particular, if, for all $\lambda \in \mathfrak{L}$,

$$
f_{\lambda}=f, \quad f: E \rightarrow \mathbb{R},
$$

we set

$$
f^{*}=\lim _{\lambda \uparrow \Lambda} f_{\lambda}
$$

$f^{*}: E^{*} \rightarrow R^{*}$ is called the natural extension of $f$. As expected, the natural extension of functions is a particular case of the extension of sets: in fact, if we identify $f$ with its graph, then $f^{*}$ is the graph of its natural extension.

\subsection{Hyperfinite sets and hyperfinite sums}

Definition 2.18. An internal set is called hyperfinite, if it is the $\Lambda$-limit of a net $\varphi: \mathfrak{L} \rightarrow \mathfrak{F}$, where $\mathfrak{F}$ is a family of finite sets.

For example, if $E \in V_{\infty}(\mathbb{R})$, the set

$$
\widetilde{E}=\lim _{\lambda \uparrow \Lambda}(\lambda \cap E)
$$

is hyperfinite. Notice that $E^{\sigma} \subset \widetilde{E} \subset E^{*}$. So we can say that every standard set is contained in a hyperfinite set. It is possible to add the elements of a hyperfinite set of numbers (or vectors) as follows: let

$$
A:=\lim _{\lambda \uparrow \Lambda} A_{\lambda}
$$


be a hyperfinite set of numbers (or vectors); then the hyperfinite sum of the elements of $A$ is defined in the following way:

$$
\sum_{a \in A} a=\lim _{\lambda \uparrow \Lambda} \sum_{a \in A_{\lambda}} a .
$$

In particular, if $A_{\lambda}=\left\{a_{1}(\lambda), \ldots, a_{\beta(\lambda)}(\lambda)\right\}$ with $\beta(\lambda) \in \mathbb{N}$, then setting

$$
\beta=\lim _{\lambda \uparrow \Lambda} \beta(\lambda) \in \mathbb{N}^{*},
$$

we use the notation

$$
\sum_{j=1}^{\beta} a_{j}=\lim _{\lambda \uparrow \Lambda} \sum_{j=1}^{\beta(\lambda)} a_{j}(\lambda) .
$$

\section{Ultrafunctions}

\subsection{Definition of ultrafunctions}

We start by introducing the notion of hyperfinite grid.

Definition 3.1. A hyperfinite set $\Gamma$ such that $\mathbb{R}^{N} \subset \Gamma \subset\left(\mathbb{R}^{N}\right)^{*}$ is called hyperfinite grid.

From now on, we assume that $\Gamma$ has been fixed once forever. Notice that, by definition, $\mathbb{R}^{N} \subseteq \Gamma$, and the following two simple (but useful) properties of $\Gamma$ can be easily proven via $\Lambda$-limits:

- for every $x \in \mathbb{R}^{N}$ there exists $y \in \Gamma \cap \mathfrak{m o n}(x)$ so that $x \neq r$;

- there exists a hyperreal number $\rho \sim 0, \rho>0$, such that $d(x, y) \geq \rho$ for every $x, y \in \Gamma, x \neq y$.

Definition 3.2. A space of grid functions is a family $\mathfrak{G}\left(\mathbb{R}^{N}\right)$ of internal functions

$$
u: \Gamma \rightarrow \mathbb{R}^{*}
$$

defined on a hyperfinite grid $\Gamma$. If $E \subset \mathbb{R}^{N}$, then $\mathfrak{G}(E)$ will denote the restriction of the grid functions to the set $E^{*} \cap \Gamma$.

Let $E$ be any set in $\mathbb{R}^{N}$. To every internal function $u \in \mathfrak{F}(E)^{*}$, it is possible to associate a grid function by the "restriction" map

$$
{ }^{\circ}: \mathfrak{F}(E)^{*} \rightarrow \mathfrak{G}(E)
$$

defined as follows:

$$
u^{\circ}(x):=u^{*}(x) \text { for all } x \in E^{*} \cap \Gamma ;
$$

moreover, if $f \in \mathfrak{F}(E)$, for short, we use the notation

$$
f^{\circ}(x):=\left(f^{*}\right)^{\circ}(x) .
$$

So every function $f \in \mathfrak{F}(E)$ can be uniquely extended to a grid function $f^{\circ} \in \mathfrak{G}(E)$.

In many problems, we have to deal with functions defined almost everywhere in $\Omega$, such as $1 /|x|$. Thus, it is useful to give a "rule" which allows to define a grid function for every $x \in \Gamma$.

Definition 3.3. If a function $f$ is defined on a set $E \subset \mathbb{R}^{N}$, we put

$$
f^{\circ}(x)=\sum_{a \in \Gamma \cap E^{*}} f^{*}(a) \sigma_{a}(x),
$$

where, for all $a \in \Gamma$, the grid function $\sigma_{a}$ is defined as follows: $\sigma_{a}(x):=\delta_{a x}$.

If $E \subset \mathbb{R}^{N}$ is a measurable set, we define the "density function" of $E$ as follows:

$$
\theta_{E}(x)=\operatorname{st}\left(\frac{m\left(B_{\eta}(x) \cap E^{*}\right)}{m\left(B_{\eta}(x)\right)}\right),
$$


where $\eta$ is a fixed infinitesimal and $m$ is the Lebesgue measure. Clearly, $\theta_{E}(x)$ is a function whose value is 1 $\operatorname{in} \operatorname{int}(E)$ and 0 in $\mathbb{R}^{N} \backslash \bar{E}$; moreover, it is easy to prove that $\theta_{E}(x)$ is a measurable function, and we have that

$$
\int \theta_{E}(x) d x=m(E)
$$

whenever $m(E)<\infty$; also, if $E$ is a bounded open set with smooth boundary, we have that $\theta_{E}(x)=\frac{1}{2}$ for every $x \in \partial E$.

Now, let $V\left(\mathbb{R}^{N}\right)$ be a vector space such that $\mathscr{D}\left(\mathbb{R}^{N}\right) \subset V\left(\mathbb{R}^{N}\right) \subset \mathscr{L}^{1}\left(\mathbb{R}^{N}\right)$.

Definition 3.4. A space of ultrafunctions $V^{\circ}\left(\mathbb{R}^{N}\right)$ modeled over the space $V\left(\mathbb{R}^{N}\right)$ is a space of grid functions such that there exists a vector space $V_{\Lambda}\left(\mathbb{R}^{N}\right) \subset V^{*}\left(\mathbb{R}^{N}\right)$ such that the map ${ }^{1}$

$$
{ }^{\circ}: V_{\Lambda}\left(\mathbb{R}^{N}\right) \rightarrow V^{*}\left(\mathbb{R}^{N}\right)
$$

is an $\mathbb{R}^{*}$-linear isomorphism. From now on, we assume that $V\left(\mathbb{R}^{N}\right)$ satisfies the following assumption: if $\Omega$ is a bounded open set such that $m_{N-1}(\partial \Omega)<\infty$ and $f \in C^{0}\left(\mathbb{R}^{N}\right)$, then

$$
f \theta_{\Omega} \in V\left(\mathbb{R}^{N}\right) .
$$

Next, we want to equip $V^{\circ}\left(\mathbb{R}^{N}\right)$ with the two main operations of calculus, the integral and the derivative.

Definition 3.5. The pointwise integral

$$
\text { ф: } V^{\circ}\left(\mathbb{R}^{N}\right) \rightarrow \mathbb{R}^{*}
$$

is a linear functional which satisfies the following properties:

(1) for all $u \in V_{\Lambda}\left(\mathbb{R}^{N}\right)$

$$
\text { 中 } u^{\circ}(x) d x=\int u(x) d x ;
$$

(2) there exists an ultrafunction $d: \Gamma \rightarrow \mathbb{R}^{*}$ such that, for all $x \in \Gamma, d(x)>0$, and for all $u \in V^{\circ}\left(\mathbb{R}^{N}\right)$,

$$
\text { 中 } u(x) d x=\sum_{a \in \Gamma} u(a) d(a) \text {. }
$$

If $E \subset \mathbb{R}^{N}$ is any set, we use the obvious notation

$$
\oint_{E} u(x) d x:=\sum_{a \in \Gamma \cap E^{*}} u(a) d(a) .
$$

A few words to discuss the above definition: Point (2) says that the pointwise integral is nothing else but a hyperfinite sum. Since $d(x)>0$, every non-null positive ultrafunction has a strictly positive integral. In particular, if we denote by $\sigma_{a}(x)$ the ultrafunctions whose value is 1 for $x=a$ and 0 otherwise, we have that

$$
\text { 中 } \sigma_{a}(x) d x=d(a) \text {. }
$$

The pointwise integral allows us to define the following scalar product:

$$
\text { 中 } u(x) v(x) d x=\sum_{a \in \Gamma} u(a) v(a) d(a) .
$$

From now on, the norm of an ultrafunction will be defined as

$$
\|u\|=\left(\oint|u(x)|^{2} d x\right)^{\frac{1}{2}} .
$$

1 We use $V^{*}(E)$ as a shorthand notation for $[V(E)]^{*}$. 
Now, let us examine point (1) of the above definition. If we take $f \in C_{\text {comp }}^{0}\left(\mathbb{R}^{N}\right)$, we have that $f^{*} \in V_{\Lambda}\left(\mathbb{R}^{N}\right)$, and hence

$$
\text { 中 } f^{\circ}(x) d x=\int f(x) d x .
$$

Thus, the pointwise integral is an extension of the Riemann integral defined on $C_{\text {comp }}^{0}\left(\mathbb{R}^{N}\right)$. However, if we take a bounded open set $\Omega$ such that $m(\partial \Omega)=0$, then we have that

$$
\int_{\Omega} f(x) d x=\int_{\bar{\Omega}} f(x) d x .
$$

However, the pointwise integral cannot have this property; in fact,

$$
\oint_{\bar{\Omega}} f^{\circ}(x) d x-\oint_{\Omega} f^{\circ}(x) d x=\oint_{\partial \Omega} f^{\circ}(x) d x>0,
$$

since $\partial \Omega \neq \emptyset$. In particular, if $\Omega$ is a bounded open set with smooth boundary and $f \in C^{0}\left(\mathbb{R}^{N}\right)$, then

$$
\begin{aligned}
\oint_{\Omega} f^{\circ}(x) d x & =\oint f^{\circ}(x) \chi_{\Omega}(x) d x \\
& =\oint f^{\circ}(x) \theta_{\Omega}^{\circ}(x) d x-\frac{1}{2} \oint f^{\circ}(x) \chi_{\partial \Omega}^{\circ}(x) d x \\
& =\int_{\Omega} f(x) d x-\frac{1}{2} \oint_{\partial \Omega} f^{\circ}(x) d x,
\end{aligned}
$$

and similarly

$$
\oint_{\bar{\Omega}} f^{\circ}(x) d x=\int_{\Omega} f(x) d x+\frac{1}{2} \oint_{\partial \Omega} f^{\circ}(x) d x ;
$$

of course, the term $\frac{1}{2} \oint f^{\circ}(x) \chi_{\partial E}(x) d x$ is an infinitesimal number and it is relevant only in some particular problems.

Definition 3.6. The ultrafunction derivative

$$
D_{i}: V^{\circ}\left(\mathbb{R}^{N}\right) \rightarrow V^{\circ}\left(\mathbb{R}^{N}\right)
$$

is a linear operator which satisfies the following properties:

(1) for all $f \in C^{1}\left(\mathbb{R}^{N}\right)$ and for all $x \in\left(\mathbb{R}^{N}\right)^{*}, x$ finite,

$$
D_{i} f^{\circ}(x)=\partial_{i} f^{*}(x)
$$

(2) for all $u, v \in V^{\circ}\left(\mathbb{R}^{N}\right)$,

$$
\oint D_{i} u v d x=-\oint u D_{i} v d x
$$

(3) if $\Omega$ is a bounded open set with smooth boundary, then for all $v \in V^{\circ}$,

$$
\oint D_{i} \theta_{\Omega} v d x=-\int_{\partial \Omega}^{*} v\left(\mathbf{e}_{i} \cdot \mathbf{n}_{E}\right) d S,
$$

where $\mathbf{n}_{E}$ is the unit outer normal, $d S$ is the $(n-1)$-dimensional measure and $\left(\mathbf{e}_{1}, \ldots, \mathbf{e}_{N}\right)$ is the canonical basis of $\mathbb{R}^{N}$;

(4) the support ${ }^{2}$ of $D_{i} \sigma_{a}$ is contained in $\mathfrak{m o n}(a) \cap \Gamma$.

2 If $u$ is an ultrafunction, the support of $u$ is the set $\{x \in \Gamma: u(x) \neq 0\}$. 
Let us comment the above definition. Point (1) implies that, for all $f \in C^{1}\left(\mathbb{R}^{N}\right)$ and for all $x \in \mathbb{R}^{N}$,

$$
D_{i} f^{\circ}(x)=\partial_{i} f(x)
$$

namely, the ultrafunction derivative coincides with the usual partial derivative whenever $f \in C^{1}\left(\mathbb{R}^{N}\right)$. The meaning of point (2) is clear; we remark that this point is very important in comparing ultrafunctions with distributions. Point (3) says that $D_{i} \theta_{\Omega}$ is an ultrafunction whose support is contained in $\partial \Omega \cap \Gamma$; it can be considered as a signed measure concentrated on $\partial \Omega$. Point (4) says that the ultrafunction derivative, as well as the usual derivative or the distributional derivative, is a local operator, namely if $u$ is an ultrafunction whose support is contained in a compact set $K$ with $K \subset \Omega$, then the support of $D_{i} u$ is contained in $\Omega^{*}$. Moreover, property (4) implies that the ultrafunction derivative is well defined in $V^{\circ}(\Omega)$ for any open set $\Omega$ by the following formula:

$$
D_{i} u(x)=\sum_{a \in \Gamma \cap \Omega^{*}} u(a) D_{i} \sigma_{a}(x) .
$$

Remark 3.7. If $u \in V^{\circ}(\Omega)$ and $\bar{u}$ is an ultrafunction in $V^{\circ}(\bar{\Omega})$ such that, for all $x \in \Omega, u(x)=\bar{u}(x)$, then, by point (3), for all $x \in \Omega^{*}$ such that $\operatorname{mon}(x) \subset \Omega^{*}$, we have that

$$
D_{i} u(x)=D_{i} \bar{u}(x) ;
$$

however, this property fails for some $x \sim \partial \Omega^{*}$. In fact, the support of $D_{i} \sigma_{a}$ is contained in $\operatorname{mon}(a) \cap \Gamma$, but not in $\{a\}$.

Theorem 3.8. There exists an ultrafunction space $V^{\circ}\left(\mathbb{R}^{N}\right)$ which admits a pointwise integral and an ultrafunction derivative as in Definitions 3.5 and 3.6.

Proof. In [2], there is a construction of a space $V_{\Lambda}\left(\mathbb{R}^{N}\right)$ which satisfies the desired properties. The conclusion follows, taking

$$
V^{\circ}\left(\mathbb{R}^{N}\right)=\left\{u^{\circ}: u \in V_{\Lambda}\right\} .
$$

\subsection{The splitting of an ultrafunction}

In many applications, it is useful to split an ultrafunction $u$ in a part $w^{\circ}$ which is the canonical extension of a standard function $w$ and a part $\psi$ which is not directly related to any classical object. If $u \in V^{\circ}(\Omega)$, we set

$$
S=\{x \in \Omega: u(x) \text { is infinite }\}
$$

and

$$
w(x)= \begin{cases}\operatorname{st}(u(x)) & \text { if } x \in \Omega \backslash S, \\ 0 & \text { if } x \in S .\end{cases}
$$

We will refer to $S$ as to the singular set of the ultrafunction $u$.

Definition 3.9. For every ultrafunction $u$, consider the splitting

$$
u=w^{\circ}+\psi,
$$

where

- $\quad w=\left.\bar{w}\right|_{\Omega \backslash S}$ and $w^{\circ}$, which is defined by Definition 3.3, is called the functional part of $u$;

- $\psi:=u-w^{\circ}$ is called the singular part of $u$.

Notice that $w^{\circ}$, the functional part of $u$, may assume infinite values for some $x \in \Omega^{*} \backslash S^{*}$, but they are determined by the values of $w$ which is a standard function defined on $\Omega$.

Example 3.10. Take $\varepsilon \sim 0$, and

$$
u(x)=\log \left(x^{2}+\varepsilon^{2}\right) .
$$


In this case,

$$
\begin{aligned}
w(x) & = \begin{cases}\log \left(x^{2}\right)=2 \log (|x|) & \text { if } x \neq 0, \\
0 & \text { if } x=0 ;\end{cases} \\
\psi(x) & = \begin{cases}\log \left(x^{2}+\varepsilon^{2}\right)-\log \left(x^{2}\right)=\log \left(1+\frac{\varepsilon^{2}}{x^{2}}\right) & \text { if } x \neq 0, \\
\log \left(\varepsilon^{2}\right) & \text { if } x=0 ;\end{cases} \\
S & :=\{0\} .
\end{aligned}
$$

We conclude this section with the following trivial proposition which, nevertheless, is very useful in applications.

Proposition 3.11. Take a Banach space $W$ such that $\mathscr{D}(\Omega) \subset W \subset L^{1}(\Omega)$. Assume that $\left\{u_{n}\right\} \subseteq V(\Omega)$ is a sequence which converges weakly in $W$ and pointwise to a function $w$; then, if we set

$$
u:=\left(\left(\lim _{\lambda \uparrow \Lambda} u_{|\lambda|}\right)^{\circ}\right),
$$

we have that

$$
u=w^{\circ}+\psi,
$$

where

moreover, if

$$
\text { 中 } \psi v d x \sim 0 \text { for all } v \in W \text {; }
$$

$$
\lim _{n \rightarrow \infty}\left\|u_{n}-w\right\|_{W}=0,
$$

then $\|\psi\|_{W} \sim 0$.

Proof. As a consequence of the pointwise convergence of $\left\{u_{n}\right\}$ to $w$, we have that, for all $a \in \Gamma, u(a) \sim w^{\circ}(a)$. In particular, for all $a \in \Gamma, \psi(a) \sim 0$. As $\Gamma$ is hyperfinite, the set $\{|\psi(a)|: a \in \Gamma\}$ has a maximum $\eta \sim 0$. Hence, for every $v \in W$, we have

$$
|\oint \psi v d x| \leq \oint|\psi||v| d x \leq \eta \text { 中 }|v| d x \sim \eta \int|v| d x \sim 0,
$$

as $\eta \sim 0$ and $\int|v| d x \in \mathbb{R}$. For the second statement, let us notice that

$$
\|\psi\|_{W}=\left\|u-w^{\circ}\right\|_{W}=\left(\lim _{\lambda \uparrow \Lambda}\left\|u_{|\lambda|}-w\right\|_{W}\right)^{\circ} \sim 0,
$$

as $\lim _{n \rightarrow \infty}\left\|u_{n}-w\right\|_{W}=0$.

An immediate consequence of Proposition 3.11 is the following:

Corollary 3.12. If $w \in L^{1}(\Omega)$, then

$$
\text { 中 } w^{\circ}(x) d x \sim \int w(x) d x \text {. }
$$

Proof. Since $V(\Omega)$ is dense in $L^{1}(\Omega)$, there is a sequence $u_{n} \in V(\Omega)$ which converges strongly to $w$ in $L^{1}(\Omega)$. Now, set

$$
u:=\left(\lim _{\lambda \uparrow \Lambda} u_{|\lambda|}\right)^{\circ}
$$

By Proposition 3.11, we have that

$$
u=w^{\circ}+\psi
$$

with $\|\psi\|_{L^{1}} \sim 0$. Then

$$
\text { 中 } u(x) d x \sim \oint w^{\circ}(x) d x .
$$

On the other hand, since $u \in V^{\circ}(\Omega)$, by Definition 3.5 (1),

$$
\begin{aligned}
\oint u(x) d x & =\int^{*} u^{\circ}(x) d x=\lim _{\lambda \uparrow \Lambda} \int u_{|\lambda|} d x \\
& \sim \lim _{n \rightarrow \Lambda} \int_{\Omega} u_{n} d x=\int w(x) d x .
\end{aligned}
$$




\subsection{The Gauss divergence theorem}

First of all, we fix the notation for the main differential operators:

- $\nabla=\left(\partial_{1}, \ldots, \partial_{N}\right)$ will denote the usual gradient of standard functions;

- $\quad \nabla^{*}=\left(\partial_{1}^{*}, \ldots, \partial_{N}^{*}\right)$ will denote the natural extension of internal functions;

- $D=\left(D_{1}, \ldots, D_{N}\right)$ will denote the canonical extension of the gradient in the sense of ultrafunctions. Next, let us consider the divergence:

- $\nabla \cdot \varphi=\partial_{1} \varphi_{1}+\cdots+\partial_{N} \varphi_{N}$ will denote the usual divergence of standard vector fields $\varphi \in\left[C^{1}\left(\mathbb{R}^{N}\right)\right]^{N}$;

- $\nabla^{*} \cdot \varphi=\partial_{1}^{*} \varphi_{1}+\cdots+\partial_{N}^{*} \varphi_{N}$ will denote the divergence of internal vector fields $\varphi \in\left[C^{1}\left(\mathbb{R}^{N}\right)^{*}\right]^{N}$;

- $D \cdot \varphi=D_{1} \varphi_{1}+\cdots+D_{N} \varphi_{N}$ will denote the divergence of vector-valued ultrafunctions $\varphi \in\left[V^{\circ}\left(\mathbb{R}^{N}\right)^{*}\right]^{N}$.

And finally, we can define the Laplace operator of an ultrafunction $u \in V^{\circ}(\Omega)$ as the only ultrafunction $\Delta^{\circ} u \in V^{\circ}(\Omega)$ such that

$$
\text { 中 } \Delta^{\circ} u v d x=-\oint D u \cdot D v d x \text { for all } v \in V_{0}^{\circ}(\bar{\Omega}) \text {, }
$$

where

$$
V_{0}^{\circ}(\bar{\Omega}):=\left\{v \in V^{\circ}(\bar{\Omega}): \text { for all } x \in \partial \Omega \cap \Gamma, v(x)=0\right\} .
$$

By Definition 3.6 (3), for any bounded open set $\Omega$ with smooth boundary,

$$
\oint D_{i} \theta_{\Omega} v d x=-\int_{\partial \Omega}^{*} v\left(\mathbf{e}_{i} \cdot \mathbf{n}_{E}\right) d S,
$$

and by Definition 3.6(2),

$$
\oint D_{i} \theta_{\Omega} v d x=-\oint D_{i} v \theta_{\Omega} d x,
$$

so that

$$
\text { 中 } D_{i} v \theta_{\Omega} d x=\int_{\partial \Omega}^{*} v\left(\mathbf{e}_{i} \cdot \mathbf{n}_{\Omega}\right) d S .
$$

Now, if we take a vector field $\varphi=\left(v_{1}, \ldots, v_{N}\right) \in\left[V^{\circ}\left(\mathbb{R}^{N}\right)\right]^{N}$, by the above identity, we get

$$
\text { 中 } D \cdot \varphi \theta_{\Omega} d x=\int_{\partial \Omega}^{*} \varphi \cdot \mathbf{n}_{\Omega} d S .
$$

Now, if $\varphi \in C^{1}$, by Definition 3.6 (1), we get the Gauss divergence theorem

$$
\int_{\Omega} \nabla \cdot \varphi d x=\int_{\partial \Omega} \varphi \cdot \mathbf{n}_{E} d S
$$

Then, (3.8) is a generalization of the Gauss theorem which makes sense for any bounded open set $\Omega$ with smooth boundary and every vectorial ultrafunction $\varphi$. Next, we want to generalize Gauss' theorem to any subset of $A \subset \mathbb{R}^{N}$. It is well known, that, for any bounded open set $\Omega$ with smooth boundary, the distributional derivative $\nabla \theta_{\Omega}$ is a vector-valued Radon measure, and we have that

$$
\left\langle\left|\nabla \theta_{\Omega}\right|, 1\right\rangle=m_{N-1}(\partial \Omega) .
$$

Then, the following definition is a natural generalization.

Definition 3.13. If $A$ is a measurable subset of $\mathbb{R}^{N}$, we set

$$
m_{N-1}(\partial \Omega):=\text { 中 }\left|D \theta_{A}^{\circ}\right| d x,
$$

and, for all $v \in V^{\circ}\left(\mathbb{R}^{N}\right)$,

$$
\oint_{\partial A} v(x) d S:=\oint v(x)\left|D \theta_{A}^{\circ}\right| d x \text {. }
$$


Remark 3.14. Notice that

$$
\oint_{\partial A} v(x) d S \neq \oint_{\partial A} v(x) d x .
$$

In fact, the left-hand term has been defined as follows:

$$
\oint_{\partial A} v(x) d S=\sum_{x \in \Gamma} v(x)\left|D \theta_{A}^{\circ}(x)\right| d(x),
$$

while the right-hand term is

$$
\oint_{\partial A} v(x) d x=\sum_{x \in \Gamma \cap \partial A^{*}} v(x) d(x) ;
$$

in particular, if $\partial A$ is smooth and $v(x)$ is bounded, $\oint_{\partial A} v(x) d x$ is an infinitesimal number.

Theorem 3.15. If $A$ is an arbitrary measurable subset of $\mathbb{R}^{N}$, we have that

$$
\text { 中 } D \cdot \varphi \theta_{A}^{\circ} d x=\oint_{\partial A} \varphi \cdot \mathbf{n}_{A}^{\circ}(x) d S,
$$

where

$$
\mathbf{n}_{A}^{\circ}(x)= \begin{cases}-\frac{D \theta_{A}^{\circ}(x)}{\left|D \theta_{A}^{\circ}(x)\right|} & \text { if } D \theta_{A}^{\circ}(x) \neq 0, \\ 0 & \text { if } D \theta_{A}^{\circ}(x)=0 .\end{cases}
$$

Proof. By Definition $3.6(3)$,

$$
\text { 中 } D \cdot \varphi \theta_{A}^{\circ} d x=- \text { 中 } \varphi \cdot D \theta_{A}^{\circ} d x \text {, }
$$

then, using the definition of $\mathbf{n}_{A}^{\circ}(x)$ and (3.9), the above formula can be written as follows:

$$
\text { 中 } D \cdot \varphi \theta_{A}^{\circ} d x=\text { 中 } \varphi \cdot \mathbf{n}_{A}^{\circ}\left|D \theta_{A}^{\circ}\right| d x=\oint_{\partial A} \varphi \cdot \mathbf{n}_{A}^{\circ} d S \text {. }
$$

\subsection{Ultrafunctions and distributions}

One of the most important properties of the ultrafunctions is that they can be seen (in some sense that we will make precise in this section) as generalizations of the distributions.

Definition 3.16. The space of generalized distributions on $\Omega$ is defined as follows:

$$
\mathscr{D}_{G}^{\prime}(\Omega)=V^{\circ}(\Omega) / N,
$$

where

$$
N=\left\{\tau \in V^{\circ}(\Omega): \text { for all } \varphi \in \mathscr{D}(\Omega), \int \tau \varphi d x \sim 0\right\} .
$$

The equivalence class of $u$ in $V^{\circ}(\Omega)$ will be denoted by $[u]_{\mathscr{D}}$.

Definition 3.17. Let $[u]_{\mathscr{D}}$ be a generalized distribution. We say that $[u]_{\mathscr{D}}$ is a bounded generalized distribution if, for all $\varphi \in \mathscr{D}(\Omega), \int u \varphi^{*} d x$ is finite. We will denote by $\mathscr{D}_{\mathrm{GB}}^{\prime}(\Omega)$ the set of bounded generalized distributions.

We have the following result.

Theorem 3.18. There is a linear isomorphism

$$
\Phi: \mathscr{D}_{\mathrm{GB}}^{\prime}(\Omega) \rightarrow \mathscr{D}^{\prime}(\Omega)
$$

such that, for every $[u] \in \mathscr{D}_{\mathrm{GB}}^{\prime}(\Omega)$ and for every $\varphi \in \mathscr{D}(\Omega)$,

$$
\left\langle\Phi\left([u]_{\mathscr{D}}\right), \varphi\right\rangle_{\mathscr{D}(\Omega)}=\operatorname{st}\left(\text { 中u} \varphi^{*} d x\right) .
$$

Proof. For the proof, see e.g. [7]. 
From now on, we will identify the spaces $\mathscr{D}_{\mathrm{GB}}^{\prime}(\Omega)$ and $\mathscr{D}^{\prime}(\Omega)$; so, we will identify $[u]_{\mathscr{D}}$ with $\Phi\left([u]_{\mathscr{D}}\right)$ and we will write $[u]_{\mathscr{D}} \in \mathscr{D}^{\prime}(\Omega)$ and

$$
\left\langle[u]_{\mathscr{D}}, \varphi\right\rangle_{\mathscr{D}(\Omega)}:=\left\langle\Phi[u]_{\mathscr{D}}, \varphi\right\rangle=\operatorname{st}\left(\text { 中u} \varphi^{*} d x\right) .
$$

If $f \in C_{\text {comp }}^{0}(\Omega)$ and $f^{*} \in[u]_{\mathscr{D}}$, then, for all $\varphi \in \mathscr{D}(\Omega)$,

$$
\left\langle[u]_{\mathscr{D}}, \varphi\right\rangle_{\mathscr{D}(\Omega)}=\operatorname{st}\left(\int^{*} u \varphi^{*} d x\right)=\operatorname{st}\left(\int^{*} f^{*} \varphi^{*} d x\right)=\int f \varphi d x .
$$

Remark 3.19. The set $V^{\circ}(\Omega)$ is an algebra which extends the algebra of continuous functions $C^{0}\left(\mathbb{R}^{N}\right)$. If we identify a tempered distribution ${ }^{3} T=\partial^{m} f$ with the ultrafunction $D^{m} f^{\circ}$, we have that the set of tempered distributions $\mathscr{S}^{\prime}$ is contained in $V^{\circ}(\Omega)$. However, the Schwartz impossibility theorem is not violated as $\left(V^{\circ}(\Omega),+, \cdot, D\right)$ is not a differential algebra since the Leibnitz rule does not hold for some pairs of ultrafunctions. See also [7].

\section{Properties of ultrafunction solutions}

The problems that we want to study with ultrafunctions have the following form: minimize a given functional $J$ on $V(\Omega)$ subjected to certain restrictions (e.g., some boundary constrictions, or a minimization on a proper vector subspace of $V(\Omega)$ ). This kind of problems can be studied in ultrafunctions theory by means of a modification of the Faedo-Galerkin method, based on standard approximations by finite-dimensional spaces. The following is a (maybe even too) general formulation of this idea.

Theorem 4.1. Let $W(\Omega) \neq \emptyset$ be a vector subspace of $V(\Omega)$. Let

$$
\begin{gathered}
\mathcal{F}=\{f: V(\Omega) \rightarrow \mathbb{R} \mid \text { for all } E \text { finite-dimensional vector subspaces of } W(\Omega), \\
\text { there exists } \left.u \in E f(u)=\min _{v \in E} f(v)\right\} .
\end{gathered}
$$

Then every $F \in \mathcal{F}^{*}$ has a minimizer in $W_{\Lambda}(\Omega)$.

Proof. Let $F=\lim _{\lambda \uparrow \Lambda} f_{\lambda}$, with $f_{\lambda} \in \mathcal{F}$ for every $\lambda \in \mathfrak{L}$. By hypothesis, for every $\lambda \in \mathfrak{L}$, there exists

$$
u_{\lambda} \in W_{\lambda}:=\operatorname{Span}(W \cap \lambda)
$$

that minimizes $f_{\lambda}$ on $W_{\lambda}$. Then $u=\lim _{\lambda \uparrow \Lambda} u_{\lambda}$ minimizes $F$ on $\lim _{\lambda \uparrow \Lambda} W_{\lambda}=W_{\Lambda}$ as, if $v=\lim _{\lambda \uparrow \Lambda} v_{\lambda} \in W_{\lambda}(\Omega)$, then, for every $\lambda \in \mathfrak{L}$, we have that $f_{\lambda}\left(v_{\lambda}\right) \leq f_{\lambda}\left(u_{\lambda}\right)$, hence,

$$
F(v)=\lim _{\lambda \uparrow \Lambda} f_{\lambda}\left(v_{\lambda}\right) \leq \lim _{\lambda \uparrow \Lambda} f_{\lambda}\left(u_{\lambda}\right)=F(u) .
$$

For applications, the following particular case of Theorem 4.1 is particularly relevant.

Corollary 4.2. Let $f(\xi, u, x)$ be coercive in $\xi$ on every finite-dimensional subspace of $V(\Omega)$ and for every $x \in \Omega$. Let $F(u):=\oint f(\nabla u, u, x) d x$. Then $F^{\circ}$ has a minimum on $V_{\Lambda}$.

Proof. Just notice that $F \in \mathcal{F}$, in the notations of Theorem 4.1.

Theorem 4.1 provides a general existence result. However, such a general result poses two questions: the first is how wild such generalized solutions can be; the second is if this method produces new generalized solutions for problems that already have classical ones.

3 We recall that, by a well-known theorem of Schwartz, any tempered distribution can be represented as $\partial^{m} f$, where $m$ is a multiindex and $f$ is a continuous function. 
The answer to these questions depends on the problem that is studied. However, regarding the second question, we have the following result, which strengthens Theorem 4.1:

Theorem 4.3. Let $F: V_{\Lambda}(\Omega) \rightarrow \mathbb{R}^{*}, F=\lim _{\lambda \uparrow \Lambda} F_{\lambda}$. For every $\lambda \in \mathfrak{L}$, let

$$
M_{\lambda}:=\left\{u \in V_{\lambda}(\Omega): F_{\lambda}(u)=\min _{v \in V_{\lambda}} F_{\lambda}(v)\right\}
$$

Assume that $\lim _{\lambda \uparrow \Lambda} M_{\lambda} \neq \emptyset$. Then

$$
M_{\Lambda}:=\left\{u \in V_{\Lambda}(\Omega): F(u)=\min _{v \in V_{\Lambda}} F(v)\right\}=\lim _{\lambda \uparrow \Lambda} M_{\lambda} \neq \emptyset
$$

Proof. $M_{\Lambda} \subseteq \lim _{\lambda \uparrow \Lambda} M_{\lambda}$ : Let $v=\lim _{\lambda \uparrow \Lambda} v_{\lambda} \in M_{\Lambda}$, and let $u=\lim _{\lambda \uparrow \Lambda} u_{\lambda} \in \lim _{\lambda \uparrow \Lambda} M_{\lambda}$. As $F(v) \leq F(u)$, there is a qualified set $Q$ such that, for every $\lambda \in Q, F_{\lambda}\left(v_{\lambda}\right) \leq F\left(u_{\lambda}\right)$. But then $v_{\lambda} \in M_{\lambda}$, for every $\lambda \in Q$, hence $v=$ $\lim _{\lambda \uparrow \Lambda} v_{\lambda} \in \lim _{\lambda \uparrow \Lambda} M_{\lambda}$.

$$
\begin{gathered}
M_{\Lambda} \supseteq \lim _{\lambda \uparrow \Lambda} M_{\lambda} \text { : Let } u=\lim _{\lambda \uparrow \Lambda} u_{\lambda} \in \lim _{\lambda \uparrow \Lambda} M_{\lambda} \text {. Let } v=\lim _{\lambda \uparrow \Lambda} v_{\lambda} \in V_{\Lambda}(\Omega) \text {. Let } \\
Q=\left\{\lambda \in \mathfrak{L}: u_{\lambda} \in M_{\lambda}\right\} .
\end{gathered}
$$

Then $Q$ is qualified and, for every $\lambda \in Q, F_{\lambda}\left(u_{\lambda}\right) \leq F_{\lambda}\left(v_{\lambda}\right)$. Therefore $F(u) \leq F(v)$, and so $u \in M_{\Lambda}$.

The following easy consequences of Theorem 4.3 hold:

Corollary 4.4. In the same notations of Theorem 4.3, let us now assume that there exists $k \in \mathbb{N}$ such that $\left|M_{\lambda}\right| \leq k$ for every $\lambda \in \mathfrak{L}$. Then $\left|M_{\Lambda}\right| \leq k$.

Proof. This holds, as the hypothesis on $\left|M_{\lambda}\right|$ trivially entails that $\left|\lim _{\lambda \uparrow \Lambda} M_{\lambda}\right| \leq k$.

Corollary 4.5. In the same notations of Theorem 4.3, let us now assume that $F=J^{*}$, where $J: V(\Omega) \rightarrow \mathbb{R}$. Let

$$
M:=\left\{v \in V(\Omega): v=\min _{w \in V(\Omega)} J(w)\right\} .
$$

Assume that $M \neq \emptyset$. Then the following facts are equivalent:

(1) $u$ is a minimizer of $F: V_{\Lambda}(\Omega) \rightarrow \mathbb{R}^{*}$.

(2) $u \in M^{*} \cap V_{\Lambda}(\Omega)$.

In particular, if $u \in M$, then $u^{*}$ minimizes $F$.

Proof. (1) $\Rightarrow(2)$ Let $u \in M$. Let $Q(u):=\{\lambda \in \mathfrak{L}: u \in \lambda\}$. Then, for every $\lambda \in Q(u)$,

$$
v \in M_{\lambda} \Longleftrightarrow J(v)=J(u) \Longrightarrow v \in M
$$

hence $M_{\lambda} \subseteq M$ for every $\lambda \in Q(u)$, which is qualified, and so $\lim _{\lambda \uparrow \Lambda} M_{\lambda} \subseteq M^{*} \cap V_{\Lambda}$, and we conclude by Theorem 4.3.

$(2) \Rightarrow(1)$ By definition,

$$
u \in M^{*} \Longleftrightarrow F(u)=\min _{v \in[V(\Omega)]^{*}} F(v),
$$

hence, if $u \in M^{*} \cap V_{\lambda}(\Omega)$, it trivially holds that $u$ minimizes $F$.

Corollary 4.6. In the same hypotheses and notations of Corollary 4.3, let us assume that $M=\left\{u_{1}, \ldots, u_{n}\right\}$ is finite. Then $v$ minimizes $F$ in $V_{\Lambda}(\Omega)$ if, and only if, there exists $u \in M$ such that $u^{*}=v$.

Proof. Just remember that $S^{\circ}=\left\{s^{*}: s \in S\right\}$ for every finite set $S$, and that

$$
M^{\sigma}=\left\{u^{*}: u \in M\right\} \subseteq V(\Omega) \subseteq V_{\Lambda}(\Omega)
$$

In general, one might not have minima, but minimization sequences could still exist. In this case, we have the following result (in which, for every $\rho \in \mathbb{R}^{*}$, we set $\operatorname{st}_{\mathbb{R}}(\rho)=-\infty$ if, and only if, $\rho$ is a negative infinite number). Notice that in the following result we are not assuming the continuity of $J$ with respect to any topology on $V(\Omega)$, in general. 
Theorem 4.7. Let $V(\Omega)$ be a Banach space, let $J: V(\Omega) \rightarrow \mathbb{R}$ and let $\inf _{u \in V(\Omega)} J(u)=m \in \mathbb{R} \cup\{-\infty\}$. The following facts hold:

(1) $J^{*}(v) \geq m$ for every $v \in V_{\Lambda}(\Omega)$.

(2) There exists $v \in V_{\Lambda}(\Omega)$ such that $\operatorname{st}_{\mathbb{R}}\left(J^{*}(v)\right)=m$.

(3) If $v \in V_{\Lambda}(\Omega)$ is a minimum of $J^{*}: V_{\Lambda}(\Omega) \rightarrow \mathbb{R}^{*}$ then $J^{*}(v) \geq \operatorname{st}_{\mathbb{R}}\left(J^{*}(v)\right)=m$.

(4) Let $\left\{u_{n}\right\}_{n \in \mathbb{N}}$ be a minimizing sequence that converges to $u \in V(\Omega)$ in some topology $\tau$. Then there exists $v \in V_{\Lambda}(\Omega)$ such that $\mathrm{st}_{\tau}(v)=u$ and $J^{*}(v) \geq \mathrm{st}_{\mathbb{R}}\left(J^{*}(v)\right)=m$. Moreover, if $w^{\circ}+\psi$ is the canonical splitting of $v$, then

- $\quad$ if $\tau$ is the topology of pointwise convergence, then $w=v$ and $w(x)=u(x)$ for every $x \in \Omega$;

- if $\tau$ is the topology of pointwise convergence a.e., then $w=v$ and $w(x)=u(x)$ a.e. in $x \in \Omega$;

- if $\tau$ is the topology of weak convergence, then $w(x)=u(x)$ for every $x \in \Omega$ and $\left\langle\psi, \varphi^{*}\right\rangle^{*} \sim 0$ for every $\varphi$ in the dual of $V(\Omega)$;

- if $\tau$ is the topology associated with a norm $\|\cdot\|$ and, moreover, $\left\{u_{n}\right\}_{n}$ converges pointwise to $u$, then $w=u$ and $\|\psi\|^{*} \sim 0$.

(5) If all minimizing sequences of $J$ converge to $u \in V(\Omega)$ in some topology $\tau$ and $v$ is a minimum of the functional $J^{*}: V_{\Lambda}(\Omega) \rightarrow \mathbb{R}^{*}$, then $\operatorname{st}_{\tau}(v)=u$ and $J^{*}(v) \geq \operatorname{st}_{\mathbb{R}}\left(J^{*}(v)\right)=m$.

Proof. (1) Let $v=\lim _{\lambda \uparrow \Lambda} v_{\lambda}$. Since $m=\inf _{u \in V(\Omega)} J(u)$, we have that $J\left(v_{\lambda}\right) \geq m$ for every $\lambda \in \Lambda$, hence $J^{*}(v) \geq m$.

(2) By (1) it suffices to show that $\operatorname{st}_{\mathbb{R}}\left(J^{*}(v)\right)=m$. Let $\left\{u_{n}\right\}_{n \in \mathbb{N}}$ be a minimizing sequence for $J$. For every $\lambda \in \mathfrak{L}$, let $v_{\lambda}:=u_{|\lambda|}$. Let $v:=\lim _{\lambda \uparrow \Lambda} v_{\lambda}$. We claim that $v$ is the desired ultrafunction.

To prove that $\operatorname{st}_{\mathbb{R}}\left(J^{*}(v)\right)=\lim _{n \rightarrow+\infty} J\left(u_{n}\right)=m$, we just have to observe that, by our definition of the net $\left\{v_{\lambda}\right\}_{\lambda}$, it follows that ${ }^{4}$

$$
\lim _{n \rightarrow+\infty} J\left(u_{n}\right)=\operatorname{st}_{\mathbb{R}}\left(\lim _{\lambda \uparrow \Lambda} J\left(v_{\lambda}\right)\right),
$$

and we conclude as $\lim _{\lambda \uparrow \Lambda} J\left(v_{\lambda}\right)=J^{*}(v)$ by definition.

(3) Let $v=\lim _{\lambda \uparrow \Lambda} v_{\lambda}$, and let $w \in V_{\Lambda}(\Omega)$ be such that $\operatorname{st}_{\mathbb{R}}\left(J^{*}(w)\right)=m$. Then $m \leq J^{*}(v)$ by (1), whilst $\operatorname{st}_{\mathbb{R}}\left(J^{*}(v)\right) \leq \operatorname{st}_{\mathbb{R}}\left(J^{*}(w)\right)=m$. Hence, $\operatorname{st}\left(J^{*}(v)\right)=m$, as desired.

(4) Let $v$ be given as in point (2). Let us show that $\operatorname{st}_{V(\Omega)}(v)=u$; let $A \in \tau$ be an open neighborhood of $u$. As $\left\{u_{n}\right\}_{n}$ converges to $u$, there exists $N>0$ such that, for every $m>N, u_{n} \in A$. Let $\mu \in \mathfrak{L}$ be such that $|\mu|>N$. Then, for every

$$
\lambda \in Q_{\mu}:=\{\lambda \in \mathfrak{L}: \mu \subseteq \lambda\}, \quad v_{\lambda} \in A,
$$

and as $Q_{\mu}$ is qualified, this entails that $v \in A^{*}$. Since this holds for every $A$ neighborhood of $u$, we deduce that $\mathrm{st}_{\tau}(v)=u$, as desired.

Now, let $u=w^{\circ}+\psi$ be the splitting of $u$.

If $\tau$ is the pointwise convergence, $\mathrm{st}_{\tau}(v)(x)=u(x)$ for every $x \in \Omega$, hence, by Definition 3.9, we have that the singular set of $u$ is empty and that $w(x)=u(x)$ for every $x \in \Omega$, as desired. A similar argument works in the case of the pointwise convergence a.e.

If $\tau$ is the weak convergence topology, then $\mathrm{st}_{\tau}(v)=u$ means that $\left\langle v, \varphi^{*}\right\rangle^{*} \sim\langle u, \varphi\rangle$ for every $\varphi$ in the dual of $V(\Omega)$. Now, let $S$ be the singular set of $u$. We claim that $S=\emptyset$. If not, let $x \in S$ and let $\varphi=\delta_{x}$. Then $\left\langle v, \varphi^{*}\right\rangle^{*}=v(x)$ is infinite, whilst $\left\langle u, \delta_{x}\right\rangle=u(x)$ is finite, which is absurd. Henceforth, for every $x \in \Omega$, we have that $\psi(x)=0$. But

$$
\langle u, \varphi\rangle \sim\left\langle v, \varphi^{*}\right\rangle^{*}=\left\langle w^{\circ}+\psi, \varphi^{*}\right\rangle^{*}=\left\langle w^{\circ}, \varphi^{*}\right\rangle^{*}+\left\langle\psi, \varphi^{*}\right\rangle^{*}=\langle w, \varphi\rangle+\left\langle\psi, \varphi^{*}\right\rangle^{*},
$$

hence, $\operatorname{st}_{\tau}(\psi)=u-w$. As $\psi(x)=0$ for all $x \in \Omega$, this means that $u(x)=w(x)$ for every $x \in \Omega$. Then

$$
\langle u, \varphi\rangle+\left\langle\psi, \varphi^{*}\right\rangle^{*}=\langle w, \varphi\rangle+\left\langle\psi, \varphi^{*}\right\rangle^{*}=\langle v, \varphi\rangle \sim\langle u, \varphi\rangle,
$$

and so $\left\langle\psi, \varphi^{*}\right\rangle^{*} \sim 0$.

4 A proof of this simple claim is given in [11, Lemma 28]. 
Finally, if $\tau$ is the strong convergence with respect to a norm $\|\cdot\|$ and $\left\{u_{n}\right\}_{n}$ converges pointwise to $u$, then, by what we proved above, we have that $v(x) \sim u(x)$ for every $x \in \Omega$, hence $u(x) \sim w(x)$ for every $x \in \Omega$, which means $u=w$ as both $u, w \in V(\Omega)$. Then $\|\psi\|=\left\|u-w^{\circ}\right\|=\left\|u-v^{\circ}\right\|+\left\|v^{\circ}-w^{\circ}\right\| \sim 0$.

(5) Let $v=\lim _{\lambda \uparrow \Lambda} v_{\lambda}$. By point (2), the only claim to prove is that $\operatorname{st}_{\tau}(v)=u$. We distinguish two cases:

Case 1: $J^{*}(v) \sim r \in \mathbb{R}$. As we noticed in point (2), it must be $r=m$. By contrast, let us assume that $\operatorname{st}_{\tau}(v) \neq u$. In this case, there exists an open neighborhood $A$ of $u$ such that the set

$$
Q:=\left\{\lambda \in \mathfrak{L}: v_{\lambda} \notin A\right\}
$$

is qualified. For every $n \in \mathbb{N}$, let

$$
Q_{n}:=\left\{\lambda \in \mathfrak{L}:\left|J\left(v_{\lambda}\right)-r\right|<\frac{1}{n}\right\} \cap Q .
$$

Every $Q_{n}$ is qualified, hence nonempty. For every $n \in \mathbb{N}$, let $\lambda_{n} \in Q_{n}$. Finally, set $u_{n}:=v_{\lambda_{n}}$. By construction, $\lim _{n \in \mathbb{N}} J\left(u_{n}\right)=m$. This means that $\left\{u_{n}\right\}_{n \in \mathbb{N}}$ is a minimizing sequence, hence, it converges to $u$ in the topology $\tau$, and this is absurd as, for every $n \in \mathbb{N}$, by construction, $u_{n} \notin A$. Henceforth, $\mathrm{st}_{\tau}(v)=u$.

Case 2: $J^{*}(v) \sim-\infty$. As we noticed in the proof of point (2), in this case $m=-\infty$. Let us assume that $\operatorname{st}_{V(\Omega)}(v) \neq u$. Then there exists an open neighborhood $A$ of $u$ such that the set

$$
Q:=\left\{\lambda \in \mathfrak{L}: v_{\lambda} \notin A\right\}
$$

is qualified. For every $n \in \mathbb{N}$, let

$$
Q_{n}=\left\{\lambda \in \mathfrak{L}: J\left(v_{\lambda}\right)<-n\right\} \cap Q
$$

and let $\lambda_{n} \in Q_{n}$. Finally, let $u_{n}:=v_{\lambda_{n}}$. Then $J\left(u_{n}\right)<-n$ for every $n \in \mathbb{N}$, hence $\left\{u_{n}\right\}_{n \in \mathbb{N}}$ is a minimizing sequence, and so it must converge to $u$. However, by construction, $u_{n} \notin A$ for every $n \in \mathbb{N}$, which is absurd.

Example 4.8. Let $\Omega=(0,1)$, let

$$
V(\Omega)=\left\{u: \Omega \rightarrow \mathbb{R} \mid u \text { is the restriction to } \Omega \text { of a piecewise } \mathcal{C}^{1}([0,1]) \text { function }\right\}
$$

and let $J: V(\Omega) \rightarrow \mathbb{R}$ be the functional

$$
J(u):=\int_{\Omega} u^{2}(x) d x+\int_{\Omega}\left(\left(u^{\prime}\right)^{2}-1\right)^{2} d x .
$$

It is easily seen that inf $u \in V(\Omega) J(u)=0$, and that the minimizing sequences of $J$ converge pointwise and strongly in the $L^{2}$ norm to 0 , but $J(0)=1$.

Let $v \in V_{\Lambda}(\Omega)$ be the minimum of $J^{*}: V_{\Lambda}(\Omega)$. From points (4) and (5) of Theorem 4.7, we deduce that $0<J^{*}(v) \sim 0$, that $\operatorname{st}_{V(\Omega)}(v)=0$ and that the canonical decomposition of $v$ is $v=0^{\circ}+\psi$, with $\psi=0$ for every $x \in \Omega$ and $\int_{\Omega^{*}}^{*} \psi^{2} d x \sim 0$. Moreover, as $J^{*}(\psi)=0$, we also have that $\int_{\Omega^{*}}^{*}\left(\left(\psi^{\prime}\right)^{2}-1\right)^{2} d x \sim 0$.

\section{Applications}

\subsection{Sign-perturbation of potentials}

The first problem that we would like to tackle by means of ultrafunctions regards the sign-perturbation of potentials.

Let us start by recalling some results recently proved by Brasco and Squassina in [13] as a refinement and extension of some classical result by Brezis and Nirenberg [14].

Let $\Omega$ be a bounded domain of $\mathbb{R}^{N}$ with $^{5} N>2$. Consider the minimization problem

$$
\mathcal{S}(a):=\inf _{u \in \mathcal{D}_{0}^{1,2}(\Omega)}\left\{\|\nabla u\|_{L^{2}(\Omega)}^{2}+\int_{\Omega} a|u|^{2} d x:\|u\|_{L^{2^{*}}(\Omega)}=1\right\},
$$

5 In [13], the authors work more in general with a $p \in(1, N)$, and consider also a fractional version of Problem 5.2; however, in this paper, we prefer to consider only the local case $p=2$. 
where $a \in L^{N / 2}(\Omega)$ is given, $2^{*}=2 N /(N-2)$,

$$
\mathcal{D}_{0}^{1,2}(\Omega):=\left\{u \in L^{2^{*}}(\Omega): \nabla u \in L^{2}(\Omega), u=0 \text { on } \partial \Omega\right\} .
$$

By Lagrange multipliers rule, minimizers of the previous problem (provided they exist) are constant sign weak solutions of

$$
\left\{\begin{aligned}
-\Delta u+a u & =\mu|u|^{2^{*}-2} u & & \text { in } \Omega, \\
u & =0 & & \text { on } \partial \Omega,
\end{aligned}\right.
$$

with $\mu=\mathcal{S}(a)$, namely

$$
\int_{\Omega} \nabla u \cdot \nabla \varphi d x+\int_{\Omega} a u \varphi d x=\mu \int_{\Omega}|u|^{2^{*}-2} u \varphi d x,
$$

for every $\varphi \in \mathcal{D}_{0}^{1,2}(\Omega)$.

The main result in [13] is the following theorem, where the standard notations

$$
a_{+}=\max \{a, 0\}, \quad a_{-}=\max \{-a, 0\}, \quad B_{R}\left(x_{0}\right)=\left\{x \in \mathbb{R}^{N}:\left|x-x_{0}\right|<R\right\}
$$

are used.

Theorem 5.1 (Brasco, Squassina). Let $\Omega \subset \mathbb{R}^{N}$ be an open bounded set. Then the following facts hold:

(1) If $a \geq 0$, then $\mathcal{S}(a)$ does not admit a solution.

(2) Let $N>4$. Assume that there exist $\sigma>0, R>0$ and $x_{0} \in \Omega$ such that

$$
a_{-} \geq \sigma, \quad \text { a.e. on } B_{R}\left(x_{0}\right) \subset \Omega \text {. }
$$

Then $\mathcal{S}(a)$ admits a solution.

(3) Let $2<N \leq 4$. For any $x_{0} \in \Omega$, for any $R>0$ s.t. $B_{R}\left(x_{0}\right) \subset \Omega$, there exists $\sigma=\sigma(R, N)>0$ such that if

$$
a_{-} \geq \sigma \text {, a.e. on } B_{R}\left(x_{0}\right),
$$

then $\mathcal{S}(a)$ admits a solution.

In [1], V. Benci studied, in the ultrafunctions setting, the following similar (simpler) problem: minimize

$$
\min _{u \in \mathfrak{M}_{p}} J(u),
$$

where

$$
J(u)=\int_{\Omega}|\nabla u|^{2} d x
$$

and

$$
\mathfrak{M}_{p}=\left\{u \in \mathcal{C}_{0}^{2}(\bar{\Omega}): \int_{\Omega}|u|^{p} d x=1\right\} .
$$

Here $\Omega$ is a bounded set in $\mathbb{R}^{N}$ with smooth boundary, $N \geq 3$ and $p>2$. In the ultrafunctions setting introduced in [1] (and with the notations of [1]), the problem takes the following form:

$$
\min _{u \in \widehat{\mathfrak{M}}_{p}} J(u)
$$

where

$$
J(u)=\int_{\Omega}^{*}|\nabla u|^{2} d x
$$

and

$$
\widetilde{\mathfrak{M}}_{p}=\left\{u \in V_{\mathcal{B}}^{2,0}(\bar{\Omega}): \int_{\Omega}^{*}|u|^{p} d x=1\right\}
$$

with $V_{\mathcal{B}}^{2,0}(\bar{\Omega})=\mathcal{B}\left[\mathcal{C}_{0}^{2}(\bar{\Omega})\right]$. 
For every $p>2$, problem (5.3) has an ultrafunction solution $\tilde{u}_{p}$ and, by setting $\widetilde{m}_{p}=J\left(\tilde{u}_{p}\right)$, one can show that

(i) if $2<p<2^{*}$, then $\widetilde{m}_{p}=m_{p} \in \mathbb{R}^{+}$and there is at least one standard minimizer $\tilde{u}_{p}$, namely $\tilde{u}_{p} \in \mathcal{C}_{0}^{2}(\bar{\Omega})$;

(ii) if $p=2^{*}$ (and $\Omega \neq \mathbb{R}^{N}$ ), then $\widetilde{m}_{2^{*}}=m_{2^{*}}+\varepsilon$, where $\varepsilon$ is a positive infinitesimal;

(iii) if $p>2^{*}$, then $\widetilde{m}_{p}=\varepsilon_{p}$, where $\varepsilon_{p}$ is a positive infinitesimal.

Our goal is to show that a similar result can be obtained for problem (5.2).

In the present ultrafunctions setting, problem (5.2) takes the following form: find

$$
\widetilde{\mathcal{S}}(a):=\inf _{u \in V_{\Lambda}(\Omega)}\left\{\int_{\left(\mathbb{R}^{N}\right)^{*}}^{*}|\nabla u|^{2} d x+\int_{\left(\mathbb{R}^{N}\right)^{*}}^{*} a|u|^{2} d x:\|u\|_{\left[L^{2^{*}}\left(\mathbb{R}^{N}\right)\right]^{*}}=1\right\},
$$

where $a \epsilon^{*}\left[L^{N / 2}(\Omega)\right]$ is given, and $V_{\Lambda}(\Omega)=\left[\mathcal{D}_{0}^{1,2}(\Omega)\right]_{\Lambda}$. With the above notations, we can prove the following:

Theorem 5.2. Let $\Omega \subset \mathbb{R}^{N}$ be an open bounded set. Then the following facts hold:

(1) For every $a \in\left[L^{N / 2}(\Omega)\right]^{*}$, there exists $u \in V_{\Lambda}(\Omega)$ that minimizes $\widetilde{\mathcal{S}}(a)$.

(2) Let $a \in\left[L^{N / 2}(\Omega)\right]$. If $u \in \mathcal{C}^{1}(\Omega) \cap \mathfrak{C}_{0}(\bar{\Omega})$ is a minimizer of problem (5.1), then $u^{*}$ is a minimizer of $\widetilde{\mathcal{S}}\left(a^{*}\right)$.

(3) If $a=0$, then $\widetilde{\mathcal{S}}(0)=S+\varepsilon$, where

$$
S:=\inf _{u \in \mathcal{D}_{0}\left(\mathbb{R}^{N}\right) \backslash\{0\}} \frac{\|\nabla u\|_{L^{2}}^{2}}{\|u\|_{L^{2^{*}}}^{2}}
$$

and

$$
\varepsilon= \begin{cases}0 & \text { if } \Omega=\mathbb{R}^{N}, \\ \text { a strictly positive infinitesimal } & \text { if } \Omega \neq \mathbb{R}^{N} ;\end{cases}
$$

moreover, if $u$ is the minimizer in $V_{\Lambda}(\Omega)$, then the functional part $w$ of $u$ is 0.

(4) Let $a \geq 0$ have an isolated minimum $x_{m}$, and let $u \in V_{\Lambda}(\Omega)$ be the minimum of problem (5.4). If $u=w^{\circ}+\psi$ is the canonical splitting of $u$, then $w=0$ and $\psi$ concentrates in $x_{m}$, in the sense that, for every $x \notin \operatorname{mon}\left(x_{m}\right)$, $\psi(x) \sim 0$. Moreover, $\left\langle\psi, \varphi^{*}\right\rangle^{*} \sim 0$ for every $\varphi$ in the dual of $V(\Omega)$.

Proof. (1) This follows from Theorem 4.3, as the functional $\|\nabla u\|_{L^{2}(\Omega)}^{2}+\int_{\Omega} a|u|^{2} d x$ admits a minimum on every finite-dimensional subspace of $V_{\Lambda}$.

(2) This follows from Corollary 4.5 .

(3) In [13, Lemma 3.1], it was proved that, if we consider problem (5.1), we have that $\mathcal{S}(0)=S$, and $\mathcal{S}(0)$ is attained in $\mathcal{D}_{0}(\Omega)$ if, and only if, $\Omega=\mathbb{R}^{N}$. Therefore, if $\Omega=\mathbb{R}^{N}$, the result follows from point (2). If $\Omega \neq \mathbb{R}^{N}$, the fact that $\widetilde{\mathcal{S}}(0)=S+\varepsilon$ follows from Theorem 4.7 (3). Moreover, all minimizing sequences $\left\{u_{n}\right\}_{n}$ converge weakly to 0 in $H^{1}$, therefore they converge strongly in $L^{2}(\Omega)$ and so they converge pointwise a.e., hence, by Theorem 4.7 (5), we deduce that, in the splitting $u=w^{\circ}+\psi$, we have that $w=0$, namely the ultrafunction solution coincides with its singular part.

(4) We start by following the approach of [13]. We let $U$ be a minimizer of

$$
\inf _{u \in \mathcal{D}_{0}^{1,2}(\Omega)} \frac{[u]_{\mathcal{D}^{1,2}}^{2}}{\|u\|_{L^{2^{*}}}^{2}}
$$

and, for every $\varepsilon>0$, let $U_{\varepsilon}(r):=\varepsilon^{\frac{2-N}{2}} U\left(\frac{r}{\varepsilon}\right)$. Let $\delta>0$ be such that $B_{\delta}\left(x_{m}\right) \subseteq \Omega$, and let $u_{\delta, \varepsilon}$ be defined as follows:

$$
u_{\delta, \varepsilon}= \begin{cases}U_{\varepsilon}(r) & \text { if } r \leq \delta, \\ U_{\varepsilon}(\delta) \frac{U_{\varepsilon}(r)-U_{\varepsilon}(\delta \Theta)}{U_{\varepsilon}(\delta)-U_{\varepsilon}(\delta \Theta)} & \text { if } \delta<r \leq \delta \Theta, \\ 0 & \text { if } r>\delta \Theta\end{cases}
$$

where $\Theta$ is a constant given in [13, Lemma 2.4]. Moreover, if $F(u):=\|\nabla u\|_{L^{2}(\Omega)}^{2}+\int_{\Omega} a|u|^{2} d x$, for $\delta_{1}, \delta_{2}$ small enough, we have that $F\left(u_{\delta_{1}, \varepsilon}\right) \leq F\left(u_{\delta_{2}, \varepsilon}\right)$. Then $\left(u_{\varepsilon, \varepsilon}\right)$ is a minimizing net (for $\varepsilon \rightarrow 0$ ), so we can use Theorem 4.7 (4). As $\left(u_{\varepsilon, \varepsilon}\right)$ converges pointwise to 0 , we obtain that $w=0$, whilst the definition of the net ensures the concentration of $\psi$ in $x_{m}$. The last statement is again a direct consequence of Theorem 4.7 (4). 
Let us notice that the above theorem shows a strong difference between the ultrafunctions and the classical case. The existence of solutions in $V_{\Lambda}(\Omega)$ is ensured independently of the sign of $a$ whilst, as discussed in [13, Section 4], the conditions on $a$ for the existence of solutions in the approach of Brasco and Squassina are essentially optimal. Of course, ultrafunction solutions might be very wild in general; their particular structure can be described in some cases, depending on $a$.

\subsection{The singular variational problem}

\subsubsection{Statement of the problem}

Let $W$ be a $C^{1}$-function defined in $\mathbb{R} \backslash\{0\}$ such that

$$
\lim _{t \rightarrow 0} W(t)=+\infty
$$

and

$$
\varlimsup_{t \rightarrow \pm \infty} \frac{W(t)}{t^{2}}=0 .
$$

We are interested in the singular problem (SP).

Naive formulation of problem SP. Find a continuous function

$$
u: \bar{\Omega} \rightarrow \mathbb{R},
$$

which satisfies the equation

$$
-\Delta u+W^{\prime}(u)=0 \quad \text { in } \Omega
$$

with the following boundary condition:

$$
u(x)=g(x) \quad \text { for } x \in \partial \Omega,
$$

where $\Omega$ is an open set such that $\partial \Omega \neq \emptyset$ and $g \in L^{1}(\partial \Omega)$ is a function different from 0 for every $x$ which changes sign, e.g. $g(x)= \pm 1$. Clearly, this problem does not have any solution in $C^{1}$. This problem could be reformulated as a kind of free boundary problem in the following way:

Classical formulation of problem SP. Find two open sets $\Omega_{1}$ and $\Omega_{2}$ and two functions

$$
u_{i}: \Omega_{i} \rightarrow \mathbb{R}, \quad i=1,2,
$$

such that all the following conditions are fulfilled:

$$
\begin{gathered}
\Omega=\Omega_{1} \cup \Omega_{2} \cup \Xi, \quad \text { where } \Xi=\bar{\Omega}_{1} \cap \bar{\Omega}_{2} \cap \Omega ; \\
-\Delta u_{i}+W^{\prime}\left(u_{i}\right)=0 \text { in } \Omega_{i}, \quad i=1,2 ; \\
u_{i}(x)=g(x) \quad \text { for } x \in \partial \Omega \cap \partial \Omega_{i}, \quad i=1,2 ; \\
\lim _{x \rightarrow \Xi} u_{i}(x)=0 ;
\end{gathered}
$$

$\Xi$ is locally a minimal surface.

Condition (5.8) is natural, since formally equation (5.5) is the Euler-Lagrange equation relative to the energy

$$
E(u)=\frac{1}{2} \int_{\Omega}\left(|\nabla u|^{2}+W(u)\right) d x,
$$

and the density of this energy diverges as $x \rightarrow \Xi$. In general this problem is quite involved since the set $\Xi$ cannot be a smooth surface and hence, it is difficult to be characterized. However, this problem becomes relatively easy if formulated in the framework of ultrafunctions. 
Let us recall that the Laplace operator of an ultrafunction $u \in V^{\circ}(\bar{\Omega})$ is defined as the only ultrafunction $\Delta^{\circ} u \in V^{\circ}(\Omega)$ such that

$$
\oint_{\Omega} \Delta^{\circ} u v d x=-\oint_{\Omega} D u \cdot D v d x, \quad \text { for all } v \in V_{0}^{\circ}(\bar{\Omega}),
$$

where

$$
V_{0}^{\circ}(\bar{\Omega}):=\left\{v \in V^{\circ}(\bar{\Omega}): \text { for all } x \in \partial \Omega \cap \Gamma, v(x)=0\right\} .
$$

Notice that, we can assert that $\Delta^{\circ} u(x)=D \cdot D(x)$ only in $x \nsim \partial \Omega^{*}$.

Ultrafunction formulation of problem $\mathbf{S P}^{6}$. Find $u \in V^{\circ}(\bar{\Omega})$ such that

$$
\begin{aligned}
u(x) & \neq 0 & & \text { for all } x \in(\bar{\Omega})^{*} \cap \Gamma, \\
-\Delta^{\circ} u+W^{\prime}(u) & =0 & & \text { for } x \in \Omega^{*} \cap \Gamma, \\
u(x) & =g^{\circ}(x) & & \text { for } x \in(\partial \Omega)^{*} \cap \Gamma .
\end{aligned}
$$

As we will see in the next section, the existence of this problem can be easily proven using variational methods.

\subsubsection{The existence result}

The easiest way to prove the existence of an ultrafunction solution of problem SP is achieved exploiting the variational structure of equation (5.11). Let us consider the extension

$$
E^{\circ}(u)=\oint_{\Omega}\left(\frac{1}{2}|D u|^{2}+W(u)\right) d x
$$

of the functional (5.9) to the space

$$
V_{g}^{\circ}(\bar{\Omega}):=\left\{u \in V^{\circ}(\bar{\Omega}): \text { for all } x \in(\partial \Omega)^{*} \cap \Gamma, u(x)=g(x)\right\} .
$$

Remark 5.3. We remark that the integration is taken over $\Omega^{*}$, but $u$ is defined in $\bar{\Omega}^{*}$. This is important, in fact, for some $x \in \Omega^{*}, x \sim \partial \Omega^{*}$, the value of $D u(x)$ depends on the value of $u$ in some point $y \in \partial \Omega^{*}, y \sim x$. This is a remarkable difference between the usual derivative and the ultrafunction derivative.

Lemma 5.4. Equation (5.11) is the Euler-Lagrange equation of the functional (5.13).

Proof. We use the expression of $\oint$ as given in Definition 3.6. As

$$
E^{\circ}(u)=\oint_{\Omega}\left(\frac{1}{2}|D u|^{2}+W(u)\right) d x,
$$

let us compute separately the variations given by $\frac{1}{2}|D u|^{2}$ and $W(u)$. As

$$
\oint_{\Omega^{*}} \frac{1}{2}|D u|^{2} d x=\sum_{a \in \Gamma \cap \Omega^{*}} \frac{1}{2}|D u(a)|^{2} d a
$$

is a quadratic form, for $v \in V_{0}^{\circ}(\bar{\Omega})$, we have that

$$
\left(\frac{d}{d u}\right)^{*}\left(\oint_{\Omega^{*}} \frac{1}{2}|D u|^{2} d x\right)[v]=\oint_{\Omega^{*}} D u D v d x=\oint_{\Omega^{*}}\left(-\Delta^{\circ} u \cdot v\right) d x .
$$

6 If $u$ is an ultrafunction and $W, W^{\prime}$, etc. are functions, for short, we shall write $W(u), W^{\prime}(u)$, etc. instead of $W^{*}(u),\left(W^{\prime}\right)^{*}(u)$, etc. 
The variation given by $W(u)$ for $v \in V_{0}^{\circ}(\bar{\Omega})$ is

$$
\left(\frac{d}{d u}\right)^{*}\left(\oint_{\Omega^{*}}(W(u) d x)\right)[v]=\left(\frac{d}{d u}\right)^{*} \sum_{a \in \Gamma \cap \Omega^{*}} W(u(a)) v(a) d a=\oint_{\Omega^{*}} W^{\prime}(u(x)) v(x) d x .
$$

Therefore, the total variation of $E^{\circ}$ is

$$
d E^{\circ}(u)[v]=\oint_{\Omega^{*}}\left(-\Delta^{\circ} u+W^{\prime}(u)\right) v d x,
$$

which proves our thesis.

The existence of an ultrafunction solution of problem SP follows from the following lemma.

Lemma 5.5. The functional (5.13) has a minimizer.

Proof. The functional $E^{\circ}(u)$ is coercive in the sense that, for any $c \in \mathbb{R}^{*}$,

$$
E^{c}:=\left\{u \in V_{g}(\bar{\Omega}): E^{\circ}(u) \leq c\right\}
$$

is hypercompact (in the sense of NSA), since $V_{g}(\bar{\Omega})$ is a hyperfinite-dimensional affine manifold. Then, since $E^{\circ}$ is hypercontinuous (in the sense of NSA), the result follows.

Regarding $\Xi$ being a minimal surface, we can prove the following:

Proposition 5.6. Let $u$ be the ultrafunction minimizer of problem (5.13), as given by Lemma 5.5. Then the sets

$$
\begin{aligned}
& \Omega_{1}=\{x \in \Omega: \text { for all } y \in \operatorname{mon}(x) \cap \Gamma, u(y)>0\}, \\
& \Omega_{2}=\{x \in \Omega: \text { for all } y \in \operatorname{mon}(x) \cap \Gamma, u(y)<0\}
\end{aligned}
$$

are open, hence

$$
\Xi:=\left\{x \in \bar{\Omega}: \text { there exist } y_{1}, y_{2} \in \mathfrak{m o n}(x) \cap \Gamma \text { such that } u\left(y_{1}\right)<0, u\left(y_{2}\right)>0\right\}
$$

is closed.

Proof. This follows from overspill? ${ }^{7}$. Let us prove it for $\Omega_{1}$. Let $x \in \Omega_{1}$. By definition of $\Omega_{1}$, for every $\varepsilon \sim 0$, we have that $u(y)<0$ for every $y \in B_{\varepsilon}(x) \cap \Gamma$. Hence, by overspill, there exists a real number $r>0$ such that $u(y)<0$ for every $y \in B_{r}^{*}(x) \cap \Gamma$. As $B_{r}^{\sigma}(x) \subset B_{r}^{*}(x) \cap \Gamma$, we deduce that the open ball $B_{r}(x) \subseteq \Omega_{1}$.

Notice that property (1) in Proposition 5.6 is a first step towards property (5.8) in the classical formulation of problem SP. It is our conjecture, in fact, that $\Xi$ is a minimal surface, at least under some rather general hypothesis. We have not been able to prove this yet, however.

Let us conclude with a remark. When studying problems like problem (5.13) with ultrafunctions, one would like to be able to generalize certain properties of elliptic equations based on the maximum principle. For example, one would expect to have the following properties:

(1) Let $\Omega$ be a bounded connected open set with smooth boundary and let $g$ be a bounded function. Then, if

$$
u=w^{\circ}+\psi
$$

is the canonical splitting of $u$, as given in Definition 3.9, we have that $w \in L^{\infty}$ and $\psi(x) \sim 0$ for every $x \in \Omega$.

7 Overspill is a well-known and very useful property in nonstandard analysis. The idea behind the version that we use here is the following: if a certain property $P(x)$ holds for every $x \sim 0$, then there must be a real number $r>0$ such that $P(x)$ holds for every $x<r$. For a proper formulation of overspill, we refer to [16]. 
(2) Let $\Omega_{1}, \Omega_{2}$ be the sets defined in Proposition 5.6. Then in $\Omega_{1} \cup \Omega_{2}$, we have

$$
\begin{aligned}
-\Delta w+W^{\prime}(w) & =0, \\
\Delta \psi(x) & \sim 0 .
\end{aligned}
$$

(3) If $a=\inf (g), b=\sup (g)$ and $W^{\prime}(t) \geq 0$ for all $t \in \mathbb{R} \backslash(a, b)$, we have that

$$
a \leq u(x) \leq b .
$$

However, in the spaces of ultrafunctions constructed in this paper, the maximum principle does not hold directly. This is due to the fact that the kernel of the derivative is, in principle, larger than the space of constants. This problem could be avoided by modifying the space of ultrafunctions. As this leads to some technical difficulties, we prefer to postpone this study to a future paper.

Funding: Marco Squassina is a member of the Gruppo Nazionale per l'Analisi Matematica, la Probabilità e le loro Applicazioni (GNAMPA) and of Istituto Nazionale di Alta Matematica (INdAM). Lorenzo Luperi Baglini has been supported by grants M1876-N35, P26859-N25 and P30821-N35 of the Austrian Science Fund FWF.

\section{References}

[1] V. Benci, Ultrafunctions and generalized solutions, Adv. Nonlinear Stud. 13 (2013), no. 2, 461-486.

[2] V. Benci, L. Berselli and C. Grisanti, The Caccioppoli ultrafunctions, Adv. Nonlinear Anal. (2017), DOI 10.1515/anona-2017-0225.

[3] V. Benci and M. Di Nasso, Alpha-theory: An elementary axiomatics for nonstandard analysis, Expo. Math. 21 (2003), no. 4, 355-386.

[4] V. Benci and L. Luperi Baglini, A model problem for ultrafunctions, in: Proceedings of the Variational and Topological Methods: Theory, Applications, Numerical Simulations, and Open Problems, Electron. J. Differ. Equ. Conf. 21 Texas State University, San Marcos (2014), 11-21.

[5] V. Benci and L. Luperi Baglini, Basic properties of ultrafunctions, in: Analysis and Topology in Nonlinear Differential Equations, Progr. Nonlinear Differential Equations Appl. 85, Birkhäuser/Springer, Cham (2014), 61-86.

[6] V. Benci and L. Luperi Baglini, Ultrafunctions and applications, Discrete Contin. Dyn. Syst. Ser. S 7 (2014), no. 4, $593-616$.

[7] V. Benci and L. Luperi Baglini, A non-archimedean algebra and the Schwartz impossibility theorem, Monatsh. Math. 176 (2015), no. 4, 503-520.

[8] V. Benci and L. Luperi Baglini, Generalized functions beyond distributions, Arab. J. Math. (Springer) 4 (2015), no. 4, 231-253.

[9] V. Benci and L. Luperi Baglini, A generalization of Gauss' divergence theorem, in: Recent Advances in Partial Differential Equations and Applications, Contemp. Math. 666, American Mathematical Society, Providence (2016), 69-84.

[10] V. Benci and L. Luperi Baglini, A topological approach to non-Archimedean mathematics, in: Geometric Properties for Parabolic and Elliptic PDE's, Springer Proc. Math. Stat. 176, Springer, Cham (2016), 17-40.

[11] V. Benci and L. Luperi Baglini, Generalized solutions in PDEs and the Burgers' equation, J. Differential Equations 263 (2017), no. 10, 6916-6952.

[12] L. Boccardo and G. Croce, Elliptic Partial Differential Equations, De Gruyter Stud. Math. 55, De Gruyter, Berlin, 2014.

[13] L. Brasco and M. Squassina, Optimal solvability for a nonlocal problem at critical growth, J. Differential Equations 264 (2018), no. 3, 2242-2269.

[14] H. Brézis and L. Nirenberg, Positive solutions of nonlinear elliptic equations involving critical Sobolev exponents, Comm. Pure Appl. Math. 36 (1983), no. 4, 437-477.

[15] J.-F. Colombeau, Elementary Introduction to new Generalized Functions, North-Holland Math. Stud. 113, North-Holland, Amsterdam, 1985.

[16] H. J. Keisler, Foundations of Infinitesimal Calculus, Prindle, Weber \& Schmidt, Boston, 1976.

[17] A. Lecke, L. Luperi Baglini and P. Giordano, The classical theory of calculus of variations for generalized smooth functions, Adv. Nonlinear Anal. (2017), DOI 10.1515/anona-2017-0150.

[18] E. Nelson, Internal set theory: A new approach to nonstandard analysis, Bull. Amer. Math. Soc. 83 (1977), no. 6, 1165-1198.

[19] M. Oberguggenberger, Generalized functions, nonlinear partial differential equations, and Lie groups, in: Geometry, Analysis and Applications (Varanasi 2000), World Scientific, Singapore (2001), 271-281.

[20] A. Robinson, Non-standard analysis, Indag. Math. 23 (1961), 432-440. 
[21] M. Sato, Theory of hyperfunctions. I, J. Fac. Sci. Univ. Tokyo. Sect. 18 (1959), 139-193.

[22] M. Sato, Theory of hyperfunctions. II, J. Fac. Sci. Univ. Tokyo Sect. 18 (1960), 387-437.

[23] M. Squassina, Existence, Multiplicity, Perturbation, and Concentration Results for a Class of Quasi-linear Elliptic Problems, Electron. J. Differ. Equ. Conf. 7, Texas State University, San Marcos, 2006. 